\title{
Effect of point defects on the electronic density of states of ScN studied by first-principles calculations and implications for thermoelectric properties
}

Sit Kerdsongpanya, Björn Alling and Per Eklund

\section{Linköping University Post Print}

\section{Tweet}

N.B.: When citing this work, cite the original article.

Original Publication:

Sit Kerdsongpanya, Björn Alling and Per Eklund, Effect of point defects on the electronic density of states of $\mathrm{ScN}$ studied by first-principles calculations and implications for thermoelectric properties, 2012, Physical Review B. Condensed Matter and Materials Physics, (86), 19.

http://dx.doi.org/10.1103/PhysRevB.86.195140

Copyright: American Physical Society http://www.aps.org/

Postprint available at: Linköping University Electronic Press http://urn.kb.se/resolve?urn=urn:nbn:se:liu:diva-87213 


\title{
Effect of point defects on the electronic density of states of $\mathrm{ScN}$ studied by first-principles calculations and implications for thermoelectric properties
}

\author{
Sit Kerdsongpanya, Björn Alling, and Per Eklund \\ Thin Film Physics Division, Department of Physics, Chemistry, and Biology (IFM), Linköping University, SE-581 83 Linköping, Sweden
}

(Received 6 September 2012; published 29 November 2012)

\begin{abstract}
We have investigated the effect of defects and impurities on the electronic density of states of scandium nitride using first-principles calculations with the generalized gradient approximation and hybrid functionals for the exchange correlation energy. Our results show that $\mathrm{Sc}$ and $\mathrm{N}$ vacancies can introduce asymmetric peaks in the density of states close to the Fermi level. We also find that the $\mathrm{N}$ vacancy states are sensitive to total electron concentration of the system due to their possibility for spin polarization. Substitutional point defects shift the Fermi level in the electronic band according to their valence but do not introduce sharp features. The energetics and electronic structure of defect pairs are also studied. By using hybrid functional calculations, a correct description of the band gap of scandium nitride is obtained. Our results envisage ways for improving the thermoelectric figure of merit of $\mathrm{ScN}$ by electronic structure engineering through stoichiometry tuning and doping.
\end{abstract}

DOI: 10.1103/PhysRevB.86.195140

PACS number(s): $71.20 . \mathrm{Nr}$

\section{INTRODUCTION}

Thermoelectric (TE) devices are solid-state devices that can directly convert thermal energy into electrical energy (or vice versa) without emission of greenhouse gases. ${ }^{1-5}$ Thermoelectric device performance depends on the temperature gradient $(\Delta T)$ and the material-specific dimensionless parameter called the thermoelectric figure of merit $(Z T)$, where $Z=S^{2} \sigma / \kappa$ and $S, \sigma$, and $\kappa$ are the Seebeck coefficient, the electrical conductivity, and the total thermal conductivity, respectively. For high performance thermoelectric devices, the materials need to be thermally stable at a large temperature gradient and have a high $Z T$ value. However, the parameters that determine $Z T$ are not independently controllable, for example, $\kappa=$ $\kappa_{\mathrm{ph}}+\kappa_{\mathrm{el}}$, where $\kappa_{\mathrm{el}}$ is electron thermal conductivity and $\kappa_{\mathrm{ph}}$ is phonon or lattice thermal conductivity. The quantities $\sigma, S, \kappa_{\mathrm{el}}$ are related to the electronic structure of the material. ${ }^{6}$ There are two general approaches for improving $Z T$ : (i) Reduce lattice thermal conductivity without reduction of $S$ and $\sigma$ which can be done by solid-solution alloying or nanostructuring, for example, nanocomposite, low-dimensional materials, superlattice, etc. ${ }^{1,7}$ and (ii) Improve the thermoelectric power factor $\left(S^{2} \sigma\right)$ with retained minimum thermal conductivity. The $S^{2} \sigma$ can be improved through doping or electronic structure engineering in which the Seebeck coefficient is increased while retaining relatively high electrical conductivity.

Mahan and Sofo ${ }^{8}$ suggested that the best thermoelectric materials require a sharp transport distribution function $\sigma(E)$ near the Fermi level $\left(E_{F}\right)$. This sharp transport distribution function will maximize the thermoelectric figure of merit for a given lattice thermal conductivity. Thus, if the transport distribution is dominated by the density of states (DOS), a narrow and sharp feature of DOS around Fermi level will maximize the power factor. Later this theoretical suggestion has been improved adding more optimization parameters, ${ }^{9}$ suggesting that an enhancement of the Seebeck coefficient occurs when the materials have an asymmetric feature of the DOS near the Fermi level. Essentially, this behavior is a refined description of the Mott formula which applies only to the Seebeck coefficient of degenerate semiconductors or metals. ${ }^{10}$ In order to have a large power factor, a transport distribution function needs to be as large as possible within the Fermi window $\left(\sim k_{B} T\right)$ to maximize electrical conductivity and as asymmetric as possible with respect to the Fermi level to enhance the Seebeck coefficient. These requirements can be attained by doping with an appropriate element to create an asymmetric sharp feature and at the same time adjust the Fermi level, or by low-dimensional materials. ${ }^{11,12}$ Moreover, these approaches also give the additional benefit of reduction of lattice thermal conductivity.

Compared to several other materials types, the transitionmetal nitrides have not been studied much for thermoelectric applications. ${ }^{13,14}$ Recently we discovered that $\mathrm{ScN}$ has a high power factor of $2.5 \mathrm{~mW} /\left(\mathrm{m} \mathrm{K}^{2}\right)$ at $800 \mathrm{~K}$ due to low metalliclike electrical resistivity $(\sim 3.0 \mu \Omega \mathrm{m})$ with a retained relatively large Seebeck coefficient of $-86 \mu \mathrm{V} / \mathrm{K} .{ }^{15}$ This power factor is very large when comparing to a state-of-the-art thermoelectric material such as $\mathrm{Bi}_{2} \mathrm{Te}_{3}$ or PbTe. ${ }^{16,17}$ Moreover, $\mathrm{ScN}$ has been shown to have suitable mechanical and electrical properties. ${ }^{18-27}$ We also reported effects on thermoelectric properties of $\mathrm{ScN}$ with small changes in the composition with the power factor changing one order of magnitude depending on, for example, oxygen, carbon, and fluorine content. The presence of impurities may influence the electronic DOS or Fermi level $\left(E_{F}\right)$. This could yield a large enhancement in the Seebeck coefficient without reduction in electrical conductivity according to the discussion above. Therefore, the effects of defects and impurities on the DOS of $\mathrm{ScN}$ need to be studied in order to understand these phenomena. Here we use a first-principles density functional theory (DFT) scheme complemented with hybrid functional calculations and a supercell approach to investigate such effects.

\section{CALCUlation DETAILS}

All calculations in this work were performed using the Vienna $a b$ initio simulation package (VASP) $\operatorname{code}^{28,29}$ using first-principles density functional calculations based on the Kohn-Sham equations ${ }^{30}$ and the projector augmented wave 
method (PAW) ${ }^{34}$ in order to calculate the electronic DOS. The generalized gradient approximation as given by PerdewBurke-Ernzerhof ${ }^{31}$ (GGA-PBE) is used as the exchangecorrelation energy in the main part of the study. In this study we have selected two supercells, which are $2 \times 2 \times 2$ conventional cubic cells consisting of 32 scandium atoms and 32 nitrogen atoms for a total of 64 atoms and $3 \times 3 \times 3$ conventional cubic cells consisting of 108 scandium atoms and 108 nitrogen atoms for a total of 216 atoms. We use the calculated lattice parameter of $\mathrm{ScN}$ from GGA-PBE giving an equilibrium value of $4.522 \AA$ in this study, which is comparable to an experimental lattice parameter of $4.50 \AA .{ }^{32,33}$ To test the numerical accuracy, the electronic DOS of $\mathrm{ScN}$ with one nitrogen vacancy system in the 64 atoms and 216 atoms supercells were used. A plane wave cutoff of 400 and $600 \mathrm{eV}$ with the $k$-point mesh of $11 \times 11 \times 11$ and $13 \times 13 \times 13$ for 64 atoms supercell and $5 \times 5 \times 5$ and $7 \times 7 \times 7$ for 216 atoms supercell, respectively, were investigated. The results of the calculations show no difference in the relevant part of the DOS when we increase the $k$-point mesh and plane wave cutoff, thus we use $11 \times 11 \times 11$ mesh for the 64 atoms supercell and $5 \times 5 \times 5$ mesh for the 216 atoms supercell with a plane wave cutoff of $400 \mathrm{eV}$ for all GGA calculation. The tetrahedral method with Blöchl correction is used in all DOS in this study. The atomic positions are allowed to relax until formation energies converge to about $10 \mathrm{meV}$ or better.

In a first step several defects have been studied on the 64 atoms supercell. They are nitrogen vacancies, and oxygen, carbon, and fluorine substitution on nitrogen site that were experimentally found in the $\mathrm{ScN}$ films. ${ }^{15}$ In addition, we also study the effect on scandium site substitution such as beryllium, magnesium, and zirconium as this could be a way to induce $p$ - or $n$-type doping of the material. Furthermore, we study scandium vacancies since this native defect could be a potentially $p$-doping agent according to a previous study. ${ }^{35}$ In this part of the investigation the concentration of impurities was 3.125 at. \%, which is derived from one substitution or one vacancy in the supercell. Next, to study low concentration and the interaction of two defects, $3 \times 3 \times 3$ supercells have been used. Here we select to study the case of dilute nitrogen vacancies (one nitrogen vacancy, $\sim 1$ at. $\%$ concentration), two nitrogen vacancies $(\sim 2$ at. $\%)$, one nitrogen vacancy with one magnesium substitution on scandium site ( 1 at. $\% \mathrm{~N}$-vac and $\sim 1$ at. $\% \mathrm{Mg}$ ), and one nitrogen vacancy with one carbon or oxygen substitution on nitrogen site $(\sim 1$ at. $\% \mathrm{~N}$ and $\sim 1$ at. $\%$ $\mathrm{C}$ or $\mathrm{O}$ ), respectively. The interaction between two defects has been done in order to find out whether they attract or repel each other and if there are particular arrangements that are favorable. Following this step the electronic density of states of the defect pairs has been calculated. In all of the calculations in this study, we allow the system to be spin polarized.

In order to investigate the consequences of the known DFT underestimation of band gaps, hybrid functional calculations using the approach suggested by Heyd, Scuseria, and Emzerhof (HSE06) ${ }^{36}$ are performed for stoichiometric $\mathrm{ScN}$ and $\mathrm{ScN}$ with one $\mathrm{N}$ vacancy in the 64-atom supercell. In this case the optimized geometries obtained in the previous GGA calculations were used and the plane wave cutoff was $300 \mathrm{eV}$.

\section{RESULTS}

\section{A. Single vacancies}

In order to understand the general effects of point defects on the electronic DOS of ScN, we will first consider the effects in $2 \times 2 \times 2$ supercell (64 total atoms). By removing or replacing one $\mathrm{Sc}$ atom or $\mathrm{N}$ atom, the defect concentration becomes 3.125 at. \%. Figure 1 shows the calculated total electronic DOS of stoichiometric $\mathrm{ScN}$ and $\mathrm{ScN}$ with vacancy defects. For stoichiometric ScN, a DOS with single point band gap is obtained instead of the experimentally measured band gap of $0.9 \mathrm{eV}^{24}$ in line with the usual GGA and LDA underestimation of band gaps. ${ }^{20,21,23,35,37}$ The use of hybrid functionals for exchange correlation energy corrects this problem (Sec. IIID). Since this investigation is directed toward possible thermoelectric applications of $\mathrm{ScN}$, from now we focus on the region around the Fermi level [area in the red square in Fig. 1(a)]. Thus Fig. 1(b) shows the total
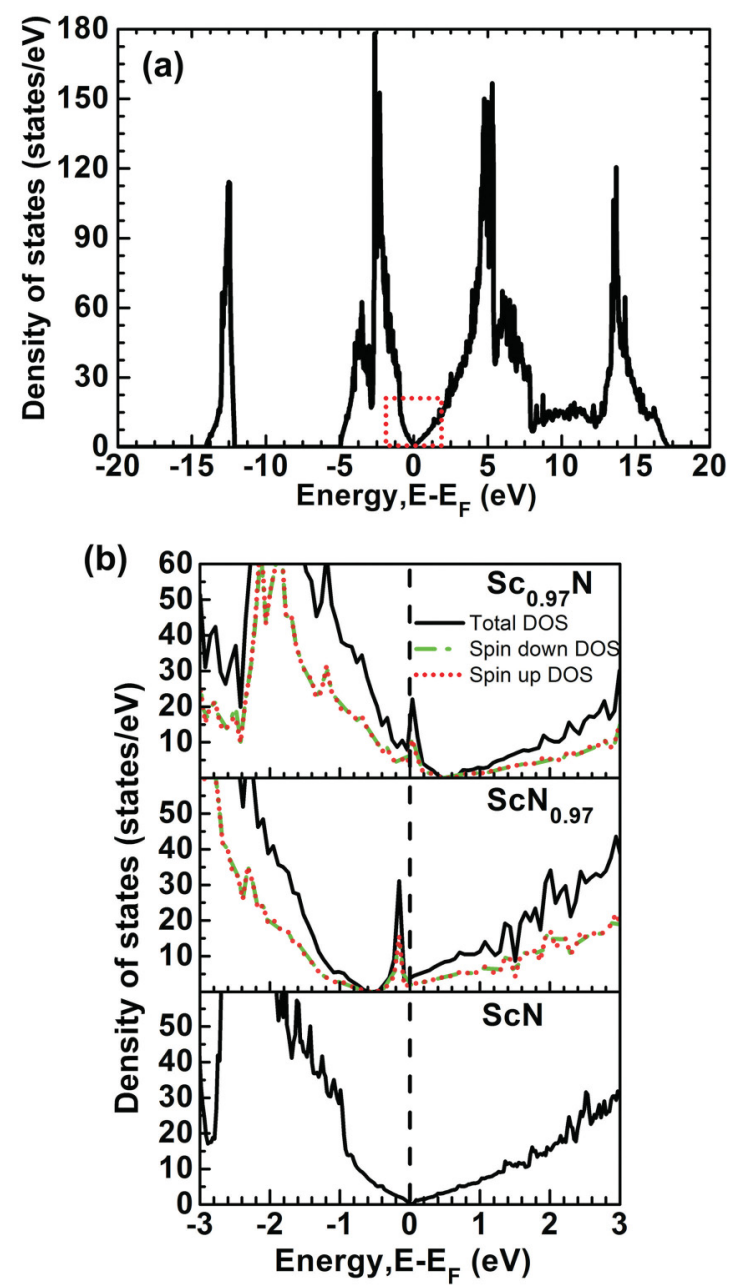

FIG. 1. (Color online) Calculated electronic density of states (DOS) of $\mathrm{ScN}$ : (a) stoichiometric $\mathrm{ScN}$ over the whole energy range and (b) enlargement of the energy range from -3 to $3 \mathrm{eV}$ marked with a red square in the total DOS. Top: $\mathrm{Sc}_{0.97} \mathrm{~N}$; middle: $\mathrm{ScN}_{0.97}$; and bottom: stoichiometric ScN. The (green) dashed and (red) dotted lines indicate the spin-up and spin-down density of states. The Fermi level which is showed by a dashed line is aligned for comparison purpose. 

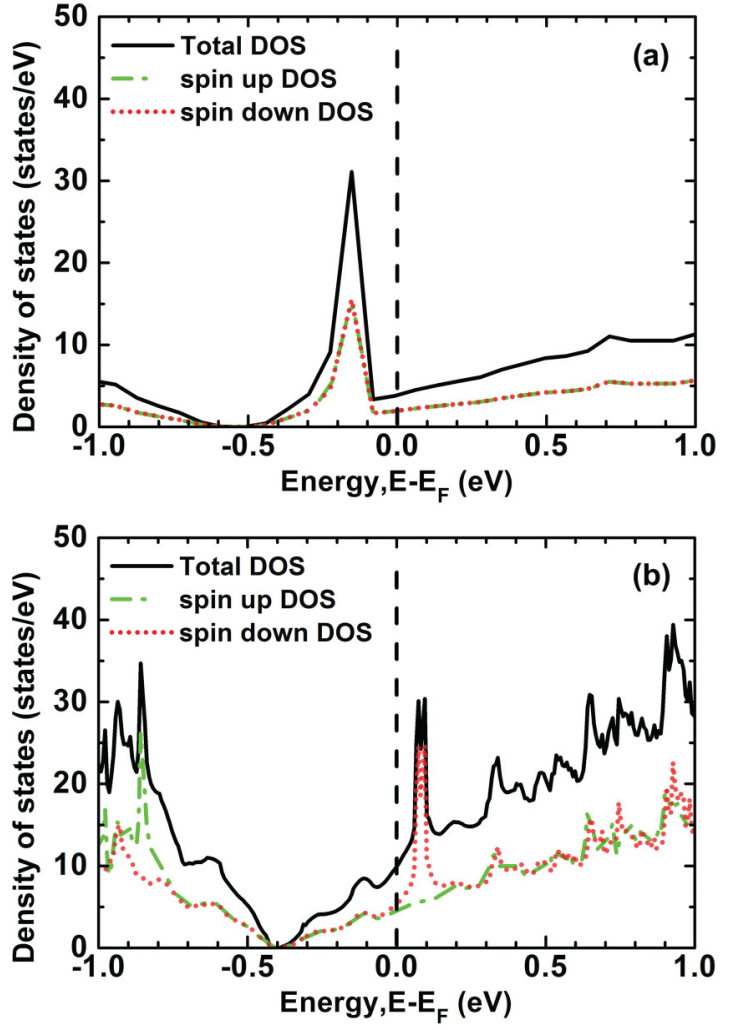

FIG. 2. (Color online) Calculated density of states of $\mathrm{ScN}$ with a single nitrogen vacancy in the energy range between -1 and $1 \mathrm{eV}$ for (a) 64 total atoms supercell and (b) 216 total atoms supercell, the (green) dashed and (red) dotted lines indicate the spin-up and spin-down density of states. Note the spin splitting in the latter case.

electronic DOS of ScN with single Sc and N vacancies. Both vacancies introduce an asymmetric peak feature close to the Fermi level. They also shift the Fermi level. A Sc vacancy moves the Fermi level downward to the valence band and a $\mathrm{N}$ vacancy lifts the Fermi level toward the conduction band. We also plot the spin-polarized density of states. In this case there is no difference between the two spin channels, thus there is no magnetic effect for any of the vacancies at these concentrations.

Next we study the electronic DOS of $\mathrm{ScN}$ for dilute nitrogen vacancies concentration. The calculation has been carried out on $3 \times 3 \times 3$ supercells with $\sim 1$ at. \% of nitrogen vacancies concentration (1 out of 108 of nitrogen atom). The formation of $\mathrm{N}$ vacancies is more probable than $\mathrm{Sc}$ vacancies in $\mathrm{ScN}$, both from the energetics as considered previously ${ }^{35}$ and from general growth dynamics considerations for nitrides, because the growth of $\mathrm{ScN}$ thin films is an out-of-equilibrium process, a fact that tends to result in more $\mathrm{N}$ vacancies than favored by thermodynamics. ${ }^{38}$ Therefore, we consider only $\mathrm{N}$ vacancies in this case. Figures 2(a) and 2(b) show the comparison of calculated DOS of $\mathrm{ScN}$ with single $\mathrm{N}$ vacancy between a small supercell (64 atoms in total) and a large supercell (216 atoms in total), respectively. The results show that there is a magnetic effect in the dilute case, visible in Fig. 2(b) as a splitting of spin-up and spin-down DOS resulting in a magnetic moment of about $0.9 \mu_{\mathrm{B}}$, where $\mu_{\mathrm{B}}$ is the Bohr magnetron.

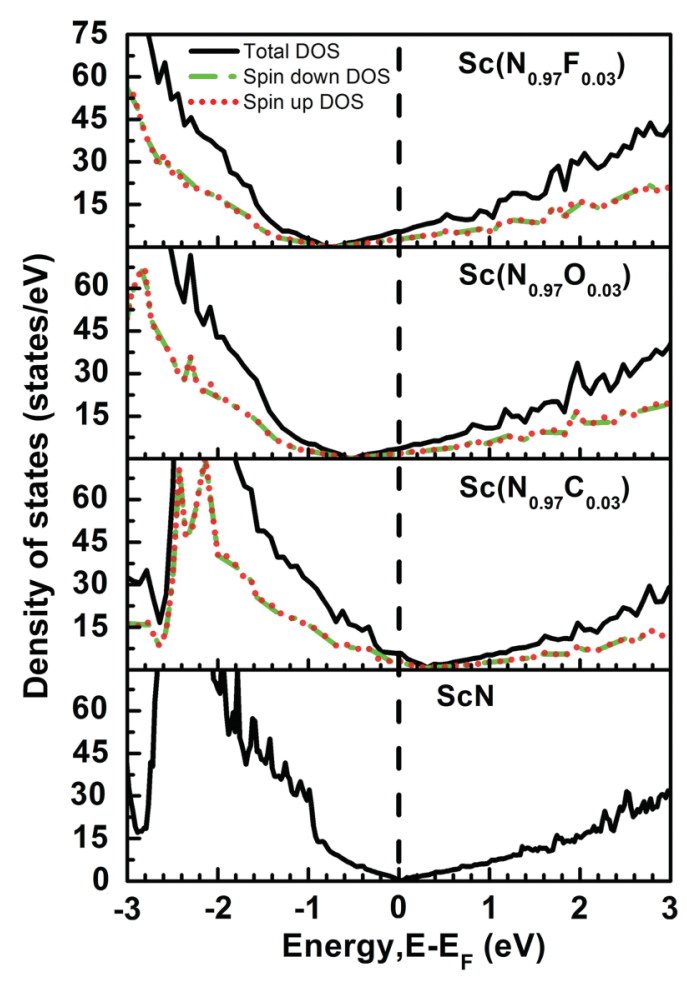

FIG. 3. (Color online) Calculated electronic density of states of doped $\mathrm{ScN}$ with energy range between -3 and $3 \mathrm{eV}$ of $\mathrm{N}$ substitutions $\left(\mathrm{ScN}_{0.97} \mathrm{C}_{0.03}, \mathrm{ScN}_{0.97} \mathrm{O}_{0.03}\right.$, and $\left.\mathrm{ScN}_{0.97} \mathrm{~F}_{0.03}\right)$.

\section{B. Single dopants or impurities}

The effects of dopants and impurities on $\mathrm{ScN}$ electronic DOS were calculated on $2 \times 2 \times 2$ supercells with 3.125 at. \% concentration of dopants and impurities. Figures 3 and 4 show the total electronic DOS of $\mathrm{ScN}$ with $\mathrm{C}, \mathrm{O}$, and $\mathrm{F}$ substituting for $\mathrm{N} \mathrm{Be}$, and $\mathrm{Mg}$ and $\mathrm{Zr}$ substituting for $\mathrm{Sc}$, respectively. None of these cases show any significant peaks appearing around the Fermi level region as in the vacancy cases. Instead, we observed a shift of the Fermi level into the conduction band of about $0.5 \mathrm{eV}$ ( $\mathrm{Zr}$ or O substituting) and $0.7 \mathrm{eV}$ (F substituting). The Fermi level is instead shifted into the valence band of about $0.4 \mathrm{eV}$ (Mg or Be substituting) and $0.2 \mathrm{eV}(\mathrm{C}$ substituting). The spin-up and spin-down DOS are identical in all those cases, therefore there are no magnetic effects when we introduce those impurities and dopants.

\section{Defect pairs}

Figure 5 shows the interaction energy between two defects calculated from the formation energies of stoichiometric $\mathrm{ScN}$, single and dual defects according to

$$
\begin{aligned}
& V_{N_{\text {vac }}-N_{\text {vac }}}=E_{2 \mathrm{~N}_{\text {vac }}}^{\mathrm{Form}}+E_{\mathrm{ScN}}^{\mathrm{Form}}-2 E_{\mathrm{N}_{\mathrm{vac}}}^{\mathrm{Form}}, \\
& V_{\mathrm{N}_{\mathrm{vac}}-\mathrm{C}_{\mathrm{sub}}, \mathrm{Mg}_{\mathrm{sub}}, \mathrm{O}_{\mathrm{sub}}=}=E_{\mathrm{N}_{\mathrm{vac}}+\mathrm{C}_{\mathrm{sub}}, \mathrm{Mg}_{\mathrm{sub}}, \mathrm{O}_{\mathrm{sub}}}^{\mathrm{Form}}+E_{\mathrm{ScN}}^{\mathrm{Form}} \\
&-E_{\mathrm{N}_{\mathrm{vac}}}^{\mathrm{Form}}-E_{\mathrm{C}_{\mathrm{sub}}, \mathrm{Mg} \mathrm{g}_{\mathrm{sub}}, \mathrm{O}_{\mathrm{sub}}}^{\mathrm{For}},
\end{aligned}
$$

where $E_{\mathrm{ScN}}^{\mathrm{Form}}$ is the formation energy of stoichiometric $\mathrm{ScN}$, $E_{\mathrm{N}_{\text {vac }}}^{\text {Form }}$ is the formation energy of $\mathrm{ScN}$ with single $\mathrm{N}$ vacancy in the supercells, $E_{\mathrm{C}_{\text {sub }}, \mathrm{Mg}_{\text {sub }}, \mathrm{O}_{\text {sub }}}^{\mathrm{Form}}$ is the formation energy of $\mathrm{ScN}$ with only either $\mathrm{C}, \mathrm{Mg}$, or $\mathrm{O}$ substitution, $E_{2 \mathrm{~N}_{\text {vac }}}^{\text {Form }}$ is 


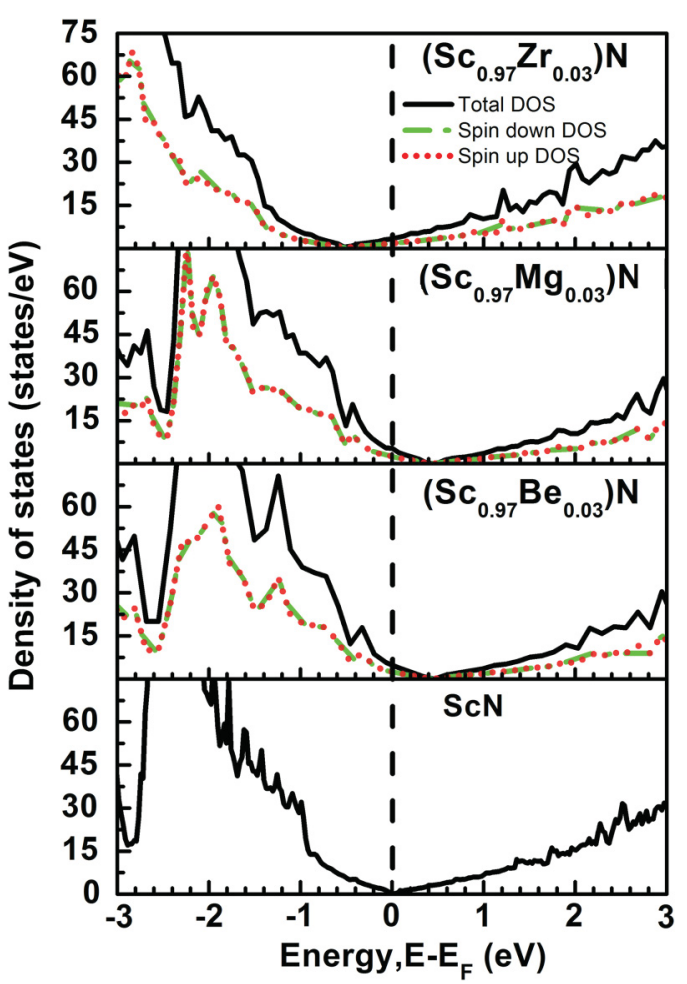

FIG. 4. (Color online) Calculated electronic density of states of doped $\mathrm{ScN}$ with energy range between -3 and $3 \mathrm{eV}$ of $\mathrm{Sc}$ substitutions ( $\mathrm{Sc}_{0.97} \mathrm{Be}_{0.03} \mathrm{~N}, \mathrm{Sc}_{0.97} \mathrm{Mg}_{0.03} \mathrm{~N}$, and $\mathrm{Sc}_{0.97} \mathrm{Zr}_{0.03} \mathrm{~N}$ ).

the formation energy of $\mathrm{ScN}$ with two $\mathrm{N}$ vacancies in the $3 \times 3 \times 3$ supercells, $E_{\mathrm{N}_{\text {vac }}+\mathrm{C}_{\text {sub }}, \mathrm{Mg}_{\text {sub }}, \mathrm{O}_{\text {sub }}}^{\mathrm{Form}}$ is the formation energy of $\mathrm{ScN}$ with single $\mathrm{N}$ vacancy and either $\mathrm{C}, \mathrm{Mg}$, or $\mathrm{O}$ substitution, and $\mathrm{V}_{\mathrm{N}_{\mathrm{vac}}-\mathrm{N}_{\mathrm{vac}}, \mathrm{O}_{\mathrm{sub}}, \mathrm{C}_{\mathrm{sub}}, \mathrm{Mg}_{\mathrm{sub}}}$ denotes the interaction potential between a $\mathrm{N}$ vacancy and a second $\mathrm{N}$ vacancy, $\mathrm{O}$ and $\mathrm{C}$ substitute on $\mathrm{N}$ site, or Mg substitute on $\mathrm{Sc}$ site, respectively. In the cases where the two defects have different effective defect charges like one $\mathrm{N}$ vacancy and $\mathrm{C}$ substitution on $\mathrm{N}$ site or Mg substitution on the Sc site [Figs. 5(a) and 5(b)], there is
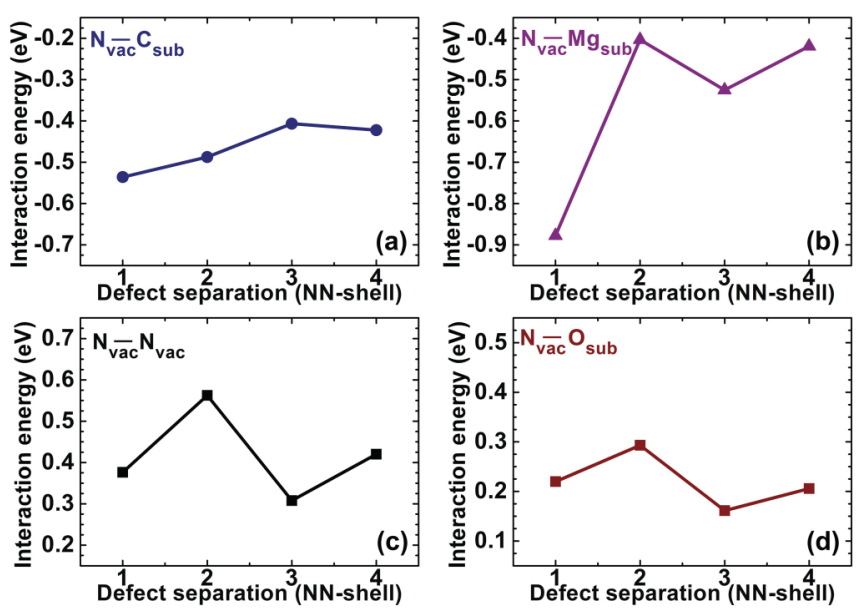

FIG. 5. (Color online) Interaction energy between two defects as a function of the defect separation (a) $\mathrm{ScN}_{0.98} \mathrm{C}_{0.01}$, (b) $\mathrm{Sc}_{0.99} \mathrm{Mg}_{0.01} \mathrm{~N}_{0.99}$, (c) $\mathrm{ScN}_{0.98}$, and (d) $\mathrm{ScN}_{0.98} \mathrm{O}_{0.01}$. The defectdefect distances corresponding to the first, second, third, and fourth nearest neighbor coordination shell are considered. a distinct attraction between the defects, favoring them to be situated at the nearest neighbor shell with a clear decrease in binding energy when the defects are moved further apart. In the case of two defects with the same effective defect charge, like two $\mathrm{N}$ vacancies or one $\mathrm{N}$ vacancy and an $\mathrm{O}$ substitution on $\mathrm{N}$ [Figs. 5(c) and 5(d)], no clear trend can be observed here at the considered distances, the lowest energy corresponding to the third coordination shell. Instead, the positive values of the interaction appear to be more determined by the total charge of the defects, which determines the Fermi level position within the calculated supercell. Based on the interaction energies, we can determine the preferred positions of all defect pairs within our 216 atoms supercell geometry. The total electronic DOS of those cases are calculated and shown in Fig. 6. All show the effect of spin splitting of $\mathrm{N}$ vacancy states and shifting of the Fermi level compared to the position when $\mathrm{ScN}$ only has $\mathrm{N}$ vacancies.

In all these studied situations, the energy gap of $\mathrm{ScN}$ is underestimated due to the well-known DFT band gap problem.

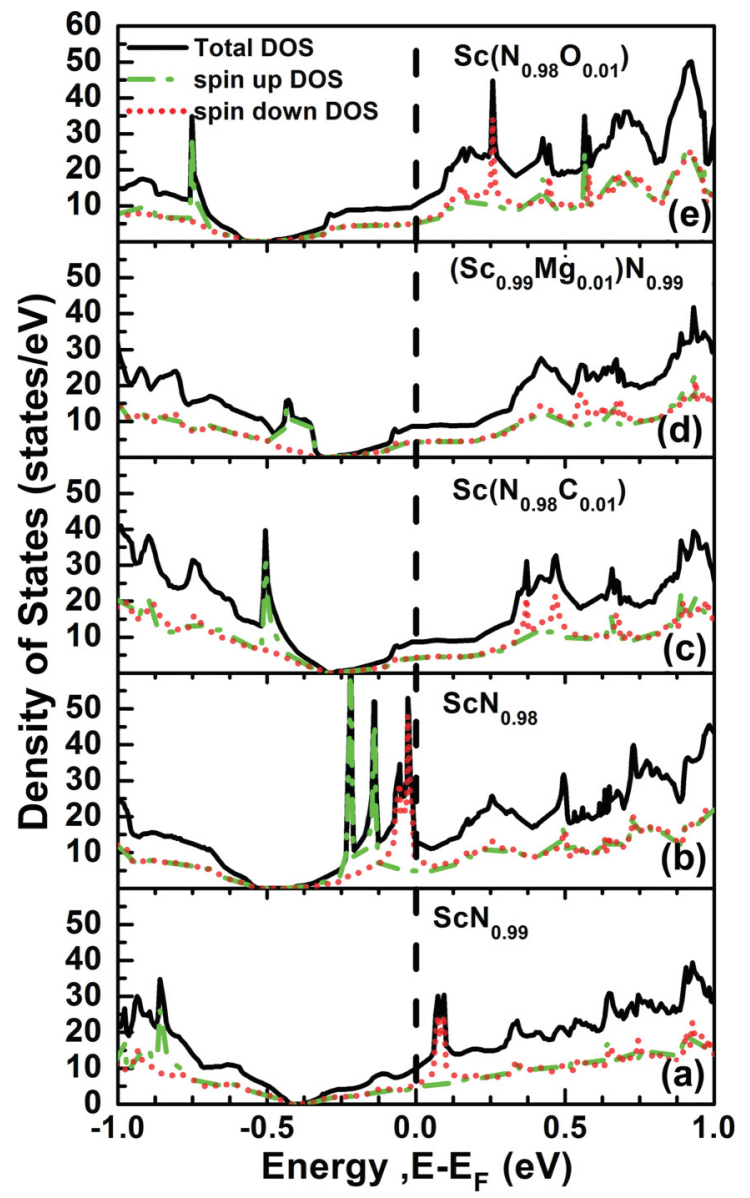

FIG. 6. (Color online) Calculated electronic density of states of energy range between -1 and $1 \mathrm{eV}$ for $\mathrm{ScN}$ with different dopants: (a) single $\mathrm{N}$ vacancy $\left(\mathrm{ScN}_{0.99}\right)$, (b) two $\mathrm{N}$ vacancies $\left(\mathrm{ScN}_{0.98}\right)$, (c) single $\mathrm{N}$ vacancy with in combination with a $\mathrm{C}$ substitution $\left(\mathrm{ScN}_{0.98} \mathrm{C}_{0.01}\right)$, (d) single $\mathrm{N}$ vacancy combined with a $\mathrm{Mg}$ substitution $\left(\mathrm{Sc}_{0.99} \mathrm{Mg}_{0.01} \mathrm{~N}_{0.99}\right)$, and (e) single $\mathrm{N}$ vacancy together with a $\mathrm{O}$ substitution $\left(\mathrm{ScN}_{0.98} \mathrm{O}_{0.01}\right)$. The (green) dashed and (red) dotted lines indicate the spin-up and spin-down density of states. The Fermi level which is showed by a dashed line is aligned for comparison purpose. 

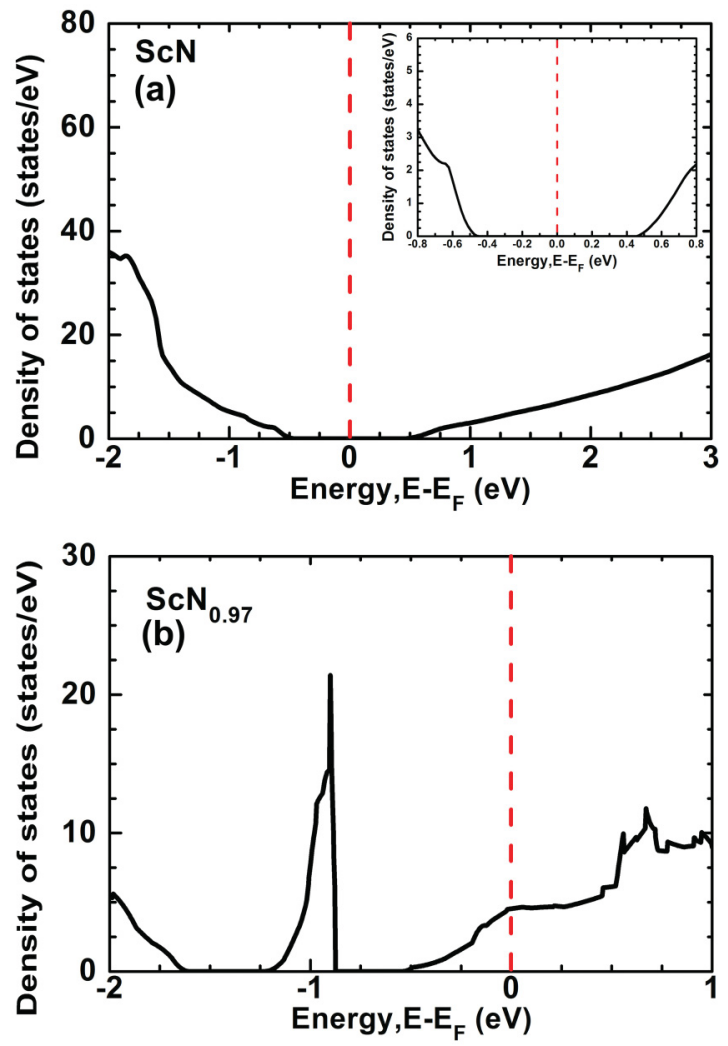

FIG. 7. (Color online) Density of states of $\mathrm{ScN}$ calculated using a hybrid functional approach: (a) stoichiometric $\mathrm{ScN}$ and (b) $\mathrm{ScN}$ with $\mathrm{N}$ vacancy $\left(\mathrm{ScN}_{0.97}\right)$, the inset in (a) shows a magnification of the region around $E_{F}$.

In order to gain insight in the importance of this factor, we consider in the next section the DOS of single $\mathrm{N}$ vacancy when we have corrected the energy gap of $\mathrm{ScN}$.

\section{Hybrid functional calculation of $\mathrm{ScN}$ electronic density of states}

Figure 7(a) shows the total DOS of $\mathrm{ScN}$ calculated by the hybrid functional HSE06 method. ${ }^{36}$ The result demonstrates the opening of an energy gap of about $0.9 \mathrm{eV}$ which is consistent with what have been reported experimentally. ${ }^{39}$ When we introduce $\mathrm{N}$ vacancy into our 64-atom supercell, the $\mathrm{N}$ vacancy introduces a sharp peak in the middle of the energy gap while the Fermi level is shifted up in a similar way as we observed in our GGA calculation [see Fig. 7(b)]. This confirms that when the energy gap of $\mathrm{ScN}$ is corrected, we still see a distinct effect of the $\mathrm{N}$ vacancy as a peak in the DOS.

\section{DISCUSSION}

In the electronic structure of stoichiometric $\mathrm{ScN}$ [Fig. 1(a)], the conduction band consists mainly of Sc $3 d$ orbitals with $t_{2 g}$ symmetry, and the valence band is formed by N $2 p$ orbital interaction with Sc $3 d$ orbitals with $e_{g}$ symmetry and Sc $4 s$ orbitals. ${ }^{40}$ The valence band is dominantly of $\mathrm{N}$ character as it has higher electronegativity. Thus, in a simplified picture, Sc can be said to donate two $4 s$ and one $3 d$ electron to fill up the $2 p$ orbital of $\mathrm{N}^{23}$ The result in Fig. 1(b) shows that removal of a Sc atom creates localized states in the $2 p$ band resulting in an asymmetric peak at the top of the valence band. Removal of one Sc atom removes three valence electrons, thus shifting the Fermi level into the valence band. In contrast, removal of a $\mathrm{N}$ atom produces an asymmetric peak at the bottom of the conduction band due to the localized states on Sc $3 d$ orbitals. In addition, by creating a $\mathrm{N}$ vacancy there will be an excess of three valence electrons from unbounded Sc electrons in the supercell. This results in a shift of the Fermi level to the conduction band. These results are in qualitative agreement with previous work that uses much higher concentrations of defects. ${ }^{35}$ The observation of these sharp features in the electronic density of states induced by vacancies could, in line with the theories discussed above, ${ }^{8}$ explain our observation that $\mathrm{ScN}$ can have a metalliclike and low electrical resistivity while still maintaining a relatively high Seebeck coefficient.

For a thermoelectric device, one needs both high $Z T$ $n$-type and $p$-type thermoelectric materials. The results of the calculations above show the possibility of obtaining either $p$-type or $n$-type $\mathrm{ScN}$ depending on how we introduce the vacancy (Sc vacancy for $p$ type and $\mathrm{N}$ vacancy for an $n$ type). Both cases can yield high $Z T$ since they generate an asymmetric peak and shift of Fermi level.

The difference in total electronic DOS of $\mathrm{ScN}$ between the two modeled vacancy concentrations observed in Figs. 2(a) and 2(b) can be understood in the following way: When we dilute the $\mathrm{N}$-vacancy concentration, we reduce the total excess electron concentration in $\mathrm{ScN}$. This reduction in electron concentration yields a decrease of the Fermi level. With lower Fermi level it becomes more favorable for the system to spin split the defect state and only occupy the spin-up part. Instead, two electrons are donated to the conduction band while empty spin-down defect states are visible right above $E_{F}$ in Fig. 2(b). As a result, a finite magnetic moment is obtained. Since the magnetic coupling between such dilute magnetic moments should be weak, this type of $\mathrm{N}$ vacancy is a paramagnetic defect. To confirm this mechanism, we did a test calculation for higher concentration of $\mathrm{N}$ vacancy by adding three well separated $\mathrm{N}$ vacancies to the 216 atoms supercell. The electronic DOS shows a similar feature as the single $\mathrm{N}$ vacancy in the 64-atom supercell that have a similar defect concentration, see Fig. 2(a). This means that higher electron concentration gives a transition from the magnetic $\mathrm{N}$ vacancies states (spin split) to nonmagnetic $\mathrm{N}$ vacancies states. These results suggest that $\mathrm{N}$ vacancy defects in $\mathrm{ScN}$ are sensitive to the total electron concentration in the system, something that could be influenced by deliberate or unintentional doping, for example, by $\mathrm{O}, \mathrm{F}$, or $\mathrm{C}$ defects or $\mathrm{Mg}$ or $\mathrm{Zr}$ doping as discussed above.

In the case of point defects (Figs. 3 and 4), $\mathrm{O}$ and $\mathrm{F}$ substitution on $\mathrm{N}$ sites, and $\mathrm{Zr}$ substitution on $\mathrm{Sc}$ site, the Fermi level moves to higher energy since $\mathrm{O}$ and $\mathrm{Zr}$ donate one electron and $\mathrm{F}$ donates two electrons. In contrast, when $\mathrm{Sc}$ is substituted by Be or $\mathrm{Mg}$ and $\mathrm{N}$ is substituted by $\mathrm{C}$, the Fermi level shifts down to the valence band. Since these elements have one electron less than $\mathrm{Sc}$ or $\mathrm{N}$ (for $\mathrm{C}$ substitution), these point defects act as acceptors and introduce holes in $\mathrm{ScN}$. The result of these calculations shows that the case of $\mathrm{C}$ substituting 
for $\mathrm{N}$ yields a smaller shift in Fermi level than $\mathrm{Mg}$ or $\mathrm{Be}$ substituting for Sc (about $0.2 \mathrm{eV}$ lower). Even though they all lack one electron. Hence, there is change in the electronic structure when we substitute $\mathrm{Mg}$ or Be on $\mathrm{Sc} . \mathrm{Mg}$ and $\mathrm{Be}$ do not have valence electrons at the $3 d$ orbital as Sc, while $\mathrm{C}$ has electronic structure similar to $\mathrm{N}$, that is, valence electrons $2 p$ orbital. This means that the electronic structure close to the defects undergo a more substantial change when $\mathrm{Mg}$ or $\mathrm{Be}$ replaces Sc as compared to when substituting $\mathrm{C}$ for $\mathrm{N}$. This yields a larger shift in Fermi level for $\mathrm{Mg}$ or Be substitutions than for $\mathrm{C}$ substituting $\mathrm{N}$.

Defect pairs with opposite effective charge tend to locate close to each other. In particular, for $\mathrm{N}$ vacancy and $\mathrm{Mg}$ substitution, as well as $\mathrm{N}$ vacancy and $\mathrm{C}$ substitution, the first coordination shell on the corresponding sublattices is a favorable configuration [see Figs. 5(a) and 5(b)]. For defects with the same effective charges, two $\mathrm{N}$ vacancies or $\mathrm{N}$ vacancy and $\mathrm{O}$ substitution, the distance dependence of the interaction is less distinct although the calculated values are positive [see Figs. 5(c) and 5(d)]. For the considered configurations here the third nearest neighbor distance is the least unfavorable configuration in those cases. For the case of $\mathrm{C}$ substitution and $\mathrm{Mg}$ substitution located next to $\mathrm{N}$ vacancy, the shape of the peaks in the DOS are different from the case of single $\mathrm{N}$ vacancy. In this case, one can also see the Fermi level slightly lowered due to reduction of electron for $\mathrm{C}$ or $\mathrm{Mg}$ doping. On the other hand, when $\mathrm{O}$ substitution on $\mathrm{N}$ site with $\mathrm{N}$ vacancy is introduced to $\mathrm{ScN}$, there is not much change on the shape of the defect peak (still sharp on spin-up and spin-down). The change in conduction band feature could be due to the replacement of $\mathrm{N}$ with $\mathrm{O}$ which may change the interaction with the $3 d$ orbitals of Sc. Two $\mathrm{N}$ vacancies and the combination of $\mathrm{O}$ substitution with single $\mathrm{N}$ vacancy give an extra electron so the Fermi level increases substantially. There is also an influence of defect-defect interactions on the DOS.

From the results of these calculations, we get significant information as to the possibility of improving the Seebeck coefficient in the $\mathrm{ScN}$ system in the following manner: Depending on the concentration of $\mathrm{N}$ vacancies, the Fermi level is either located above the sharp feature (high $\mathrm{N}$ vacancy concentrations) or below the unoccupied spin-down part (paramagnetic low concentration of $\mathrm{N}$ vacancies). This implies the possibility of tailoring the electron concentration and Fermi level position with respect to those sharp features using either lower valence ( $\mathrm{Mg}$ on $\mathrm{Sc}$ site, $\mathrm{C}$ on $\mathrm{N}$ site) or higher valence ( $\mathrm{Zr}$ on $\mathrm{Sc}$ site, $\mathrm{O}$ or $\mathrm{F}$ on $\mathrm{N}$ site) dopants in addition to the $\mathrm{N}$ vacancy concentration. It may thus be possible to enhance the Seebeck coefficient and power factor as shown in the case of two $\mathrm{N}$ vacancies in our supercell, corresponding to about 2 at. \% of $\mathrm{N}$ vacancy in $\mathrm{ScN}$, the Fermi level is located at the edge of an asymmetric peak (see Fig. 6). Moreover, introducing, for example, $\mathrm{Mg}$ or $\mathrm{Zr}$ in a $\mathrm{ScN}$ matrix may yield phonon scattering by point defects which can lower thermal conductivity. So the doping of $\mathrm{ScN}$ in the presence of $\mathrm{N}$ vacancies seems to be an interesting system to study for high thermoelectric figure of merit. Note that point defects can influence electron scattering yielding reduction of electrical conductivity, an effect that should be considered together with attempts to maximizing electrical conductivity and locating the Fermi level on a sharp DOS region for enhancement of the Seebeck coefficient.

Based on all of these results it would, in principle, be possible to perform transport property calculations of this system. However, the underestimated band gap of $\mathrm{ScN}$ can give errors (see, e.g., Ref. 41), where the calculated Seebeck coefficient was of the wrong order of magnitude for $\mathrm{ScN}$ and even the wrong sign for $\mathrm{ZrN}$. Thus we consider hybridfunctional calculations in order to correct the band gap problem and confirm that results that we obtained in GGA still appear when we have correct the band gap for $\mathrm{ScN}$.

The hybrid functional calculation showed that the most drastic effect on the DOS found in the GGA calculations, the asymmetric sharp peak induced by $\mathrm{N}$ vacancy, is still present even when the band gap is corrected to $0.9 \mathrm{eV}$ (see Fig. 7). We currently do not readily have the computational capacity to use the hybrid functional approach to calculate the DOS of defects in the larger supercell (e.g., 216 atoms), taking magnetism into account. Nevertheless, our hybrid functional results suggest that the same type of behavior as in the GGA case should be present also with the corrected energy gap, with a competition between spin-polarized and non-spin-polarized defect states with a dependence on the total electron concentration. However, a prediction of quantitative values of which particular concentrations of vacancies as well as dopants that are optimal for improving the thermoelectric power factor is beyond the reach of the present approaches.

Based on these results, we suggest that one could indeed improve the thermoelectric power factor of $\mathrm{ScN}$ by manipulating the ScN DOS and the Fermi level by deliberate introduction of defects and dopants. Even though it is difficult to quantitatively determine the exact optimal concentrations of vacancies and dopants, the general strategy can be outlined based on the present results. By selective choices of dopants, the electron concentration could be tailored to move the Fermi level close to the defect peak with a possibility for an enhancement of the Seebeck coefficient. Introducing defects and dopants gives another benefit in term of lattice thermal conductivity reduction due to phonon scattering at point defect.

\section{CONCLUSION}

We have investigated the effect of defects and impurities on the electronic DOS of $\mathrm{ScN}$ by a density functional theory (DFT) scheme and a supercell approach. Our results demonstrate that $\mathrm{Sc}$ and $\mathrm{N}$ vacancies can introduce asymmetric peaks in the DOS of ScN. Small additions of substitutional impurities and dopants shift the Fermi level in the electronic band. $\mathrm{F}$ and $\mathrm{O}$ in $\mathrm{N}$ sites and $\mathrm{Zr}$ in Sc sites shift the Fermi level toward the conduction band (electron donor), while $\mathrm{C}$ in $\mathrm{N}$ sites and $\mathrm{Be}$ and $\mathrm{Mg}$ in $\mathrm{Sc}$ sites shift the Fermi level down to the valence band (electron deficient) without the introduction of any sharp features. For dilute $\mathrm{N}$ vacancy concentration, there is spin splitting effect occurring at the nitrogen defect levels.

The hybrid functional has been used to correct the energy gap of ScN. The results show that there is an opening of energy gap of about $0.9 \mathrm{eV}$ around the Fermi level. While we introduce $\mathrm{N}$ vacancy, it gives a sharp peak in the middle of the energy gap with the Fermi level and is shifted similar to GGA calculation. 
Our results indicate the possibility of obtaining a large Seebeck coefficient without reducing the electrical conductivity by moving the Fermi level near the location of the vacancy peak in the DOS by introducing an appropriate dopant with the presence of $\mathrm{N}$ vacancies, which could yield an enhancement of thermoelectric power factor $\left(S^{2} \sigma\right)$. Moreover, these dopants could help reduce the lattice thermal conductivity due to phonon scattering at the point defects. Thus, this study shows that there is a promising pathway to obtain high thermoelectric figure of merit in $\mathrm{ScN}$ due to the possibility of enhancement of the Seebeck coefficient and reduction of lattice thermal conductivity.

\section{ACKNOWLEDGMENTS}

The authors would like to acknowledge funding from the Swedish Research Council (VR) through Grants No. 621-2009-5258 and No. 621-2011-4417 and the Linnaeus Strong Research Environment LiLi-NFM, the Swedish Foundation for Strategic Research (Ingvar Carlsson Award 3), and the Linköping Center in Nanoscience and technology (CeNano). The calculations were performed using computer resources provided by the Swedish national infrastructure for computing (SNIC) at the National Supercomputer Centre (NSC).
*Corresponding author: sitke@ifm.liu.se

${ }^{1}$ C. J. Vineis, A. Shakouri, A. Majumdar, and M. G. Kanatzidis, Adv. Mater. 22, 3970 (2010).

${ }^{2}$ J. R. Sootsman, D. Y. Chung, and M. G. Kanatzidis, Angew. Chem., Int. Ed. 48, 8616 (2009).

${ }^{3}$ G. J. Snyder and E. S. Toberer, Nat. Mater. 7, 105 (2008).

${ }^{4}$ F. J. DiSalvo, Science 285, 703 (1999).

${ }^{5}$ T. M. Tritt, Annu. Rev. Mater. Res. 41, 433 (2011).

${ }^{6}$ A. Shakouri, Annu. Rev. Mater. Res. 41, 399 (2011).

${ }^{7}$ J.-F. Li, W.-S. Liu, L.-D. Zhao, and M. Zhou, NPG Asia Mater 2, 152 (2010).

${ }^{8}$ G. D. Mahan and J. O. Sofo, Proc. Natl. Acad. Sci. USA 93, 4436 (1996).

${ }^{9}$ Z. Fan, H.-Q. Wang, and J.-C. Zheng, J. Appl. Phys. 109, 073713 (2011).

${ }^{10}$ N. F. Mott and H. Jones, The Theory of the Properties of Metals and Alloys (Dover, New York, 1958).

${ }^{11}$ J. P. Heremans, V. Jovovic, E. S. Toberer, A. Saramat, K. Kurosaki, A. Charoenphakdee, S. Yamanaka, and G. J. Snyder, Science 321, 554 (2008).

${ }^{12}$ M. S. Dresselhaus, G. Chen, M. Y. Tang, R. G. Yang, H. Lee, D. Z. Wang, Z. F. Ren, J. P. Fleurial, and P. Gogna, Adv. Mater. 19, 1043 (2007).

${ }^{13}$ C. X. Quintela, F. Rivadulla, and J. Rivas, Appl. Phys. Lett. 94, 152103 (2009).

${ }^{14}$ M. Zebarjadi, Z. Bian, R. Singh, A. Shakouri, R. Wortman, V. Rawat, and T. Sands, J. Electron. Mater. 38, 960 (2009).

${ }^{15}$ S. Kerdsongpanya et al., Appl. Phys. Lett. 99, 232113 (2011).

${ }^{16}$ Y. Deng, H.-M. Liang, Y. Wang, Z.-W. Zhang, M. Tan, and J.-L. Cui, J. Alloy. Compd. 509, 5683 (2011).

${ }^{17}$ Y.-L. Pei and Y. Liu, J. Alloy. Compd. 514, 40 (2012).

${ }^{18}$ D. Gall, I. Petrov, N. Hellgren, L. Hultman, J. E. Sundgren, and J. E. Greene, J. Appl. Phys. 84, 6034 (1998).

${ }^{19}$ D. Gall, M. Städele, K. Järrendahl, I. Petrov, P. Desjardins, R. T. Haasch, T. Y. Lee, and J. E. Greene, Phys. Rev. B 63, 125119 (2001).
${ }^{20}$ W. R. L. Lambrecht, Phys. Rev. B 62, 13538 (2000).

${ }^{21}$ C. Stampfl, W. Mannstadt, R. Asahi, and A. J. Freeman, Phys. Rev. B 63, 155106 (2001).

${ }^{22}$ G. Travaglini, F. Marabelli, R. Monnier, E. Kaldis, and P. Wachter, Phys. Rev. B 34, 3876 (1986).

${ }^{23}$ A. Qteish, P. Rinke, M. Scheffler, and J. Neugebauer, Phys. Rev. B 74, 245208 (2006).

${ }^{24}$ H. A. Al-Brithen, A. R. Smith, and D. Gall, Phys. Rev. B 70, 045303 (2004).

${ }^{25}$ J. P. Dismukes, W. M. Yim, J. J. Tietjen, and R. E. Novak, RCA Rev. 31, 680 (1970)

${ }^{26}$ J. M. Gregoire, S. D. Kirby, M. E. Turk, and R. B. van Dover, Thin Solid Films 517, 1607 (2009).

${ }^{27}$ J. M. Gregoire, S. D. Kirby, G. E. Scopelianos, F. H. Lee, and R. B. van Dover, J. Appl. Phys. 104, 074913 (2008).

${ }^{28}$ G. Kresse and J. Furthmüller, Phys. Rev. B 54, 11169 (1996).

${ }^{29}$ G. Kresse and D. Joubert, Phys. Rev. B 59, 1758 (1999).

${ }^{30}$ W. Kohn and L. J. Sham, Phys. Rev. 140, A1133 (1965).

${ }^{31}$ J. P. Perdew, K. Burke, and M. Ernzerhof, Phys. Rev. Lett. 77, 3865 (1996).

${ }^{32}$ W. Lengauer, J. Solid State Chem. 76, 412 (1988).

${ }^{33}$ D. Gall, I. Petrov, P. Desjardins, and J. E. Greene, J. Appl. Phys. 86, 5524 (1999).

${ }^{34}$ P. E. Blöchl, Phys. Rev. B 50, 17953 (1994).

${ }^{35}$ M. G. Moreno-Armenta and G. Soto, Comp. Mater. Sci. 40, 275 (2007).

${ }^{36}$ A. V. Krukau, O. A. Vydrov, A. F. Izmaylov, and G. E. Scuseria, J. Chem. Phys. 125, 224106 (2006).

${ }^{37}$ A. van de Walle and G. Ceder, Phys. Rev. B 59, 14992 (1999).

${ }^{38}$ L. Hultman, U. Helmersson, S. A. Barnett, J. E. Sundgren, and J. E. Greene, J. Appl. Phys. 61, 552 (1987).

${ }^{39}$ H. A. H. Al-Brithen, E. M. Trifan, D. C. Ingram, A. R. Smith, and D. Gall, J. Cryst. Growth 242, 345 (2002).

${ }^{40}$ W. A. Harrison and G. K. Straub, Phys. Rev. B 36, 2695 (1987).

${ }^{41}$ B. Saha, J. Acharya, T. D. Sands, and U. V. Waghmare, J. Appl. Phys. 107, 033715 (2010). 